\title{
A Hospital Based Study of Association of Chronic Spontaneous Urticaria with Autologous Serum Skin Test
}

\author{
Giri U1', Kayastha BMM², Shakya NB ${ }^{3}$
}

Department of Dermatology, ${ }^{1}$ National Academy of Medical Sciences, Kathmandu, Nepal. ${ }^{2}$ Patan Academy of Health Sciences, Patan, Nepal, ${ }^{3}$ Nepalese Army Institute of Healthy Sciences

\begin{abstract}
Introduction: Chronic Spontaneous Urticaria (CSU), is spontaneous occurrence of wheals for more than 6 weeks. CSU patients with autoreactivity as indicated by positive Autologous Serum Skin Test (ASST) have severe disease requiring higher doses of antihistamines and even immunomodulatory agents.

Objective: The objective of this study was to observe the association between CSU and ASST.

Materials and Methods: Over a period of one year, $104 \mathrm{CSU}$ patients were enrolled in the study. Autologous serum skin test was done using $0.05 \mathrm{ml}$ of patient's serum, with $0.05 \mathrm{ml}$ of histamine as positive control and normal saline as negative control. Prevalence of positive ASST in CSU patients was determined. Clinical and demographic characteristics were compared in both ASST positive and negative patients.

Results: The ASST was positive in 68 patients (65.4\%). Mean age \pm SD of the patients in ASST positive group was 33.78 \pm 14.38 years versus $35.64 \pm 14.47$ years in ASST negative group $(p=0.533$ ). Male: Female ratio in ASST group was $1: 2.7$ versus 1:1.1 in ASST negative group ( $p=0.033$ ). Patients with positive ASST had significantly longer duration of wheals $(p=0.002)$, generalized distribution $(p=0.020)$ and high rmean urticaria activity score, 4.66 versus 3.28 in ASST negative patients, $p<0.001$. Angioedema was more common in ASST positive patients $(p<0.001)$.

Conclusion: ASST was significantly positive in CSU patients and more common in females. ASST positive patients had more severe clinical manifestations and it can be a simple test to detect severe form of CSU.

Key words: Angioedema; Chronic Urticaria; Histamine; Skin Tests
\end{abstract}

\section{Introduction}

U ticaria is one of the most frequent skin diseases affecting up to one in four in the total population once in their lifetime. Urticaria is characterized by pruritic wheal and flare type skin reaction with or without angioedema that usually persists for less than 24 hours ${ }^{1}$ Chronic Spontaneous Urticaria (CSU), is characterized by the spontaneous occurrence of urticaria for more than six weeks independent of external stimuli. ${ }^{2}$ All age groups (peak age $20-40$ years) can develop a CSU with the point prevalence of CSU being 0.5 to $1 \% .^{2}$

Financial disclosure: None.

Conflict of interest to disclosure: None declared.

Address of Correspondence

Dr. Uma Giri

Consultant Dermatologist

National Academy of Medical Sciences

Kathmandu, Nepal.

E-mail: frenship54@hotmail.com
Many of CSU patients have positive ASST (Autologous serum skin test), which involves demonstration of papular and erythematous reaction to intradermal injection of autologous serum. The test detects active IgG autoantibodies against the high affinity IgE receptor ( $F c \in R \mid \alpha)$ or IgE and has been an important in vivo test in patients with CSU. ${ }^{3-5}$ The frequency of ASST versus responses for adult CSU patients in published studies ranges from $4.1 \%$ to $76.5 \%$. A positive ASST

Submitted: $10^{\text {th }}$ September 2019

Accepted: $30^{\text {th }}$ October 2019

Published: $7^{\text {th }}$ October 2020

How to cite this article

Giri U, Kayastha BMM, Shakya NB. A hospital based study of Association of chronic spontaneous urticaria with autologous serum skin test. Nepal Journal of Dermatology Venereology and Leprology 2020;18(1):9-14. https://doi.org/10.3126/njdvl. v18i1.30172.

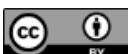

Licensed under CC BY 4.0 International License which permits use, distribution and reproduction in any medium, provided the original work is properly cited. 
has only moderate specificity as a marker for these functional autoantibodies, but a negative ASST has a high negative predictive value. ${ }^{6}$ ASST positive patients have severe disease, higher urticarial activity score, require higher dose of antihistamine and even immunomodulatory agents for the control of disease. ${ }^{7-10}$ So the assessment of the CSU patients by ASST can help in the management. The work was done with the objective of demonstrating the association of ASST positivity amongst patient of CSU presenting in a tertiary hospital in Kathmandu.

\section{Materials and Methods}

This prospective cross-sectional observational study was done in the outpatient department of dermatology of National Academy of Medical Sciences, Bir hospital, Kathmandu from March 2013 to February 2014.

Following the ethical clearance from the Institutional Review Board (IRB), all consecutive patients presenting with spontaneous occurrence of wheals with or without angioedema for more than six weeks' duration independent of external stimuli were included in the study. Children $\leq 14$ years, pregnant and lactating women, physical urticarial patient, patient taking antihistamine till two to three days, doxepin till two weeks before the test and patient taking any corticosteroid at the time of examination, patient taking immunosuppressant drug, severely ill patients, patient with Human Immune Deficiency Virus Infection were excluded from the study.

A detail history regarding the characteristics and possible eliciting factors of chronic spontaneous urticaria were taken. Data was collected using a semistructured questionnaire. Details included time of onset of CSU, frequency and duration of wheals, duration of individual wheal in hours, duration of disease in months, size and distribution of wheals; associated angioedema; associated subjective symptoms of lesion, e.g. itch, pain; personal and family history of urticaria and atopy; previous or present allergies, infections, internal diseases; psychiatric diseases; surgical implantation, Gastric/intestinal problems, induction by physical agents or exercise, observed correlation to food, use of drugs (NSAIDs, injections, immunizations, hormones, laxatives, suppositories, ear and eye drops and alternative remedies); relationship to menstrual cycle; smoking habits; type of work; stress; quality of life related to urticaria (sleep and emotional impact); previous therapy and response to therapy and history of other systemic disease including autoimmune disease. Symptoms and signs were graded on the basis of Urticarial activity score, ranging from 0 to 3 which are indicative of mild, moderate or intense disease. ${ }^{1}$ Examination was done.

On the same day Autologous Serum Skin Test was performed and the result of the test was observed. All these data were recorded in a pre-designed pro-forma. Two millilitres of venous blood was collected in a sterile plastic tube and allowed to clot at room temperature for $30 \mathrm{~min}$. The serum was centrifuged at $2000 \mathrm{rpm}$ for $15 \mathrm{~min}$. The ASST was performed using $1 \mathrm{ml}$ sterile insulin syringe without dead space using $0.05 \mathrm{ml}$ fresh undiluted serum. A superficial intradermal injection was made by introducing the bevel of the needle uppermost and aiming to raise a palpable bleb of fluid within the papillary dermis. The ASST response was validated by performing a positive histamine control, by intradermal injection of $0.5-1 \mu \mathrm{g}(0.05 \mathrm{ml}$ of $0.01 \mathrm{mg} /$ $\mathrm{ml}$ ) histamine. For emergency due to severe reaction injection epinephrine $(0.01 \mathrm{mg} / \mathrm{kg}$ to a maximum of $1 \mathrm{mg}$ ) along with antihistamine (chlorpheniramine) were kept ready. As a negative control skin test, $0.05 \mathrm{ml}$ sterile normal saline was injected intradermally using the same method as per serum. For each intradermal injection separate sterile insulin syringe was used. Serum induced wheal which had average diameter of $\geq 1.5 \mathrm{~mm}$ as compared to the saline-induced wheal at 30 min was recorded as Positive ASST.

Data entry was done using excel, analysed using SPSS 17. Demographic data, duration of CSU in months, age of onset of CSU, frequency of urticarial attacks, duration, distribution, site and size of wheals, association of angioedema, systemic symptoms, history of drug use, atopy and food allergy were compared between ASST positive and negative groups. Descriptive statistics was used to summarise data for comparison between ASST positive and negative groups. Chi square test was used for categorical variables. Independent t test and Mann Whitney $U$ test was used for continuous variables. p-value of $<.05$ was considered significant.

\section{Results}

One hundred and four CSU patients (age 15-86 years) out of which 69 were females and 35 were males were studied. Autologous Serum Skin Test was positive in 68 patients $(65.4 \%)$ and negative in 36 patients (34.6\%). The mean age was $33.78 \pm 14.38$ months in ASST positive patients and $35.64 \pm 14.47$ months in ASST negative patients, $p$ value 0.533 . The mean age of disease onset was $30.78 \pm 14.753$ months in ASST positive group versus $33.97 \pm 14.358$ months in ASST negative groups, $p$ value 0 . 292.The male to female ratio among ASST positive group was 1:2.7 versus 1:1.1 among ASST negative group with a $p$ value 0.033 . 
The median duration of disease was 12 months 2,300) in ASST group versus $10(2,144)$ in ASST negative group, p-value 0.846 . Fifty-two (76\%) patients in ASST positive group had daily attack compared to 23 (63\%) in ASST negative group, p value 0.291. Forty patients (59\%) in ASST positive group had duration of wheals more than 6 hours versus 8 (32\%) in patients without positive ASST, $p$ value 0.002 . The mean value for the size of serum induced wheals was $10.14 \pm 2.53$ in ASST positive group versus $7.83 \pm 3.07$ in ASST negative group with a $p$-value $<.001$. Twenty nine ASST positive patients (42.6\%) had $>50$ wheals in 24 hours compared to four (11\%) ASST negative patients, $p$ value $<0.001$. Thirty five (51.5\%) patients with ASST positivity had generalised wheal versus $10(27.8 \%)$ in ASST negative patients. Extremities were most common site of involved in ASST positive patients versus face and trunk in ASST negative patients (figure1).

Severity of pruritus was defined by disturbance of daily activities. Intense pruritus was observed in 32 (39.7\%) ASST positive patients versus 2 (5.6\%) ASST negative patients, $p$ value $<0.001$. Sixty two ASST positive patients (91.2\%) had urticarial severity score of 4-6 versus 15 (41\%) in ASST negative patients with a $p$ value of $<0.001$. Forty-one ASST positive patients (60.3\%) had angioedema versus 13 (36.1\%) ASST negative patients, $p$ value 0.019 . Thirty two (30.8\%) patients had angioedema of both lips and eyes. Twenty six (38.2\%) of the 32 patients with involvement of eyes and lips were ASST positive.

Eleven ASST positive (16.2\%) patients had h/o atopy. Twelve out of 68 ASST positive patients (17.6\%) had positive family history versus 11 (30.6\%) ASST negative patients, $\mathrm{p}$ value 0.131 . Food allergy was observed in $16(23.4 \%)$ ASST positive patients versus 6 (16.7\%) ASST negative patients, $p$ value 0.415 . Seventeen of the $104(16 \%)$ patients had associated systemic diseases. Hypertension was the most common associated disease. Twelve (68\%) ASST positive patients had systemic disease versus 5 (13.9\%) ASST negative patients, $p$ value 0.622 (table 2 ).

Table 1: Clinical parameters based on ASST positivity

\begin{tabular}{|c|c|c|c|}
\hline & ASST positive & ASST negative & P value \\
\hline Mean Age (years) & 33.78 & 35.64 & 0.533 \\
\hline Female & 50 & 19 & \multirow{2}{*}{0.033} \\
\hline Male & 18 & 17 & \\
\hline $\begin{array}{l}\text { Mean duration of the } \\
\text { disease (months) }\end{array}$ & 12 & 10 & 0.846 \\
\hline Age of onset & 30.78 & 33.97 & 0.292 \\
\hline \multicolumn{3}{|l|}{ Number of lesion } & \multirow{4}{*}{$<0.001$} \\
\hline$<20$ in 24 hours & 6 & 20 & \\
\hline $20-50$ & 33 & 12 & \\
\hline$>50$ & 29 & 4 & \\
\hline \multicolumn{3}{|l|}{ Frequency of attack } & \multirow{4}{*}{0.291} \\
\hline Daily & 52 & 23 & \\
\hline 1-3/week & 14 & 10 & \\
\hline 4-6/week & 2 & 3 & \\
\hline \multicolumn{3}{|l|}{ Duration of Wheals } & \multirow{4}{*}{0.002} \\
\hline$<6$ hours & $28(41.2 \%)$ & $28(77.8 \%)$ & \\
\hline 6 to 12 hours & 35 (51.5\%) & $7(19.4 \%)$ & \\
\hline 13 to $>24$ hours & $5(7.4 \%)$ & $1(2.8 \%)$ & \\
\hline \multicolumn{3}{|l|}{ UAS } & \multirow{3}{*}{$<0.001$} \\
\hline $0-3$ & 6 & 21 & \\
\hline $4-6$ & 62 & 15 & \\
\hline \multicolumn{3}{|l|}{ Site of lesion } & \multirow{3}{*}{$<0.020$} \\
\hline Localized & 33 & 26 & \\
\hline Generalized & 35 & 10 & \\
\hline \multicolumn{3}{|l|}{ Angioedema } & \multirow{3}{*}{0.019} \\
\hline Present & 41 & 13 & \\
\hline Absent & 27 & 23 & \\
\hline
\end{tabular}


Table 2: Risk factors based on ASST positivity

\begin{tabular}{|l|c|c|c|}
\hline Risk factors & ASST positive & ASST negtive & P value \\
\hline H/O atopy & 11 & 9 & 0.277 \\
Yes & 57 & 27 & \\
No & 16 & 6 & 0.415 \\
\hline H/O food allergy & 52 & 30 & \\
Yes & & 11 & 0.131 \\
No & 12 & 25 & 0.62 \\
\hline Family history of atopy & 56 & 5 & 0.432 \\
Yes & 12 & 8 & 0.935 \\
NO & 20 & 14 & 0.13 \\
\hline H/O Systemic disease & 27 & 9 & \\
YES & 9 & 3 & 0.79 \\
\hline H/O Stress & 1 & 12 & \\
\hline YeS & 19 & 2 & \\
\hline Disturbance of sleep & 5 & 22 & \\
\hline H/O surgery & 44 & & \\
\hline H/O menstrual cycle & & & \\
\hline H/O Drug use & & & \\
NSAIDS & & & \\
Contraceptive & & & \\
None & & & \\
\hline
\end{tabular}

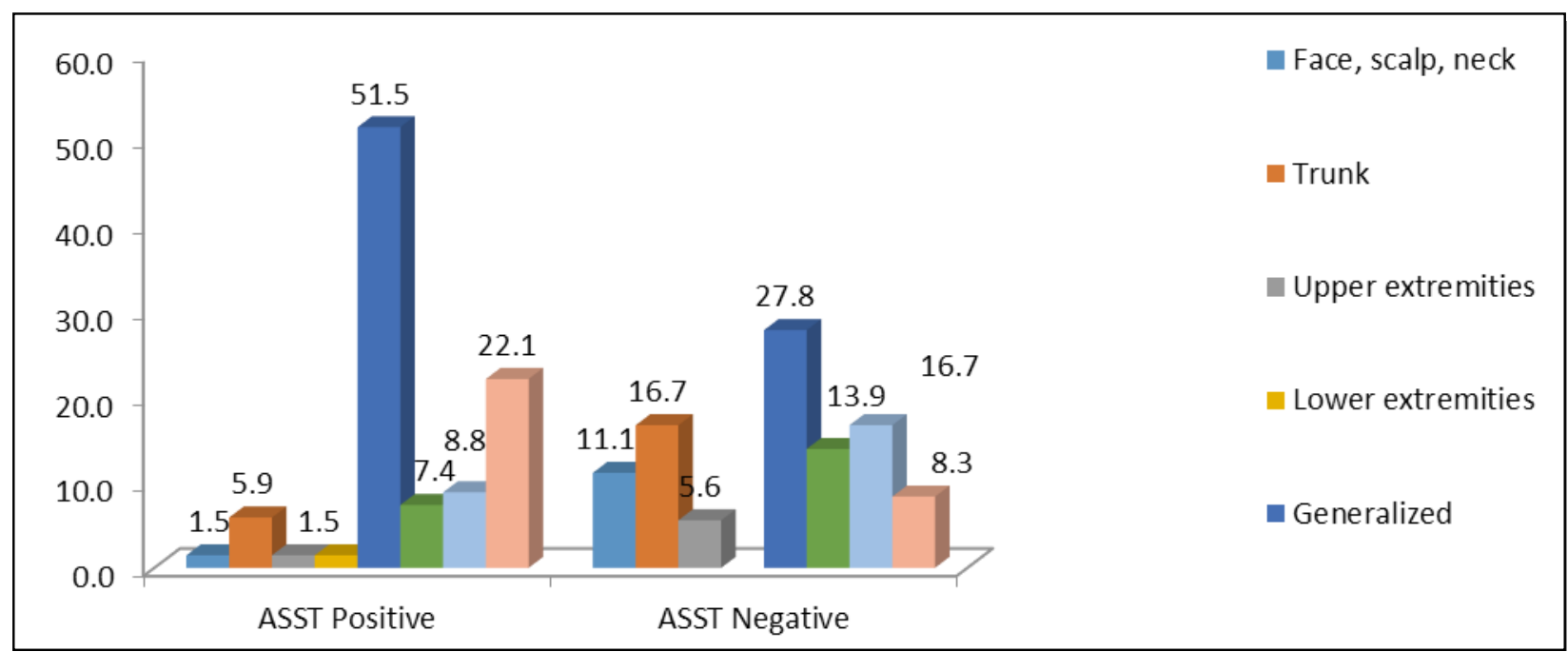

Figure 1: Site of involvement in patients with and without positive ASST

\section{Discussion}

Chronic spontaneous urticaria is common clinical condition characterised by development of wheals, angioedema or both for more than 6 weeks independent of external stimuli. The rapid search into aetiology fails to demonstrate any aetiology. The evaluation for autoimmunity by autologous serum skin test can help predict the clinical course and management of the cases as ASST positive cases require prolonged therapy. The present study has evaluated patients with chronic spontaneous urticaria (CSU) by autologous serum skin testing and compared the demography and clinical features of patients with positive and negative ASST results. The proportion of patients with CSU who showed a positive reaction to ASST was $65.4 \%$ which is comparable with earlier reports. In Chinese CSU patients positive autologous serum skin tests (ASST positive) were observed in $66.9 \%{ }^{11}$ Italian patients with CIU showed a positive ASST in $67 \% .{ }^{12}$ Whereas in India the frequency of positive ASST varied in different studies from $26.67 \%$ to $58.75 \% .{ }^{13,14}$ The variation in prevalence according to the ethnic group of population suggests a genetic background for the disease. Since 
ASST has a sensitivity of $70 \%$ and a specificity of $80 \%$, positive test is indicatory but not diagnostic of an autoimmune basis for chronic urticaria. Confirmation is needed by in vitro testing of the patient's serum for anti-FceR1 $\alpha$ or anti-IgE autoantibody. ${ }^{15}$

Though all age groups can be affected by CSU, the peak incidence is seen between 20 and 40 years. $^{2}$ Our study shared similar findings with the majority of patients in the age group 25-34 years. The mean age of presentation was not different amongst ASST positive and negative group which was consistent to several other studies. ${ }^{7-10}$ The incidence of CSU in women is twice than that in men. ${ }^{2}$ In our study also the number of female was predominant ( 69 females and 35 males). The male female ratio among ASST positive group was 1:2.7 whereas it was 1:1.1 among ASST negative group. Our study showed that the prevalence of a positive ASST was significantly higher in women $(73.50 \%)$ than in men $(26.50 \%)$. This is comparable with the studies by Asero, which showed higher ASST positivity in females than in males (76\% versus $35 \%) .{ }^{16}$

In our study the median duration of disease in ASST positive group was 12 months while that of ASST negative group was 10 months, but the difference was not statistically significant $(p=0.846)$. This finding corresponds to the study by Kulthana et al where median duration of disease in ASST positive and negative group was 14 and 13 months, respectively $(p=0.9) .^{17}$

Urticaria severity score, a score to measure severity of urticaria was higher in ASST positive patients in different studies. Our study also showed similar results with the UAS score being higher in ASST positive group(UAS $4.66 \pm 0,940 p=<0.0091$ ). Our finding is consistent with an Indian study by Vohra et al. ${ }^{10}$

CSU is characterised by prolonged wheals. The duration of wheals can vary in patients with and without ASST positivity. Present study showed ASST positive patients showing longer duration than the ASST negative. ASST positive patients had statistically significant long lasting wheals compared to ASST negative patients $(p=0.002)$. Similar finding was noted in the studies done by George et al and Zhong et al. ${ }^{11}$

The concomitant angioedema was more common in ASST positive patients compared to ASST negative patients which was similar to finding depicted in an Chinese and Indian study. ${ }^{11,14}$ There was insignificant difference between two groups regarding history of atopy, family history of allergy and food allergy. This finding was in agreement with that reported by AlHamamy et al and Krupashankar et al. ${ }^{14,18}$

\section{Conclusion}

Autologous serum skin test (ASST) was associated with significant proportion (65.4\%) of patients with CSU.ASST positivity was observed higher with female gender and young age. Patients with positive ASST had higher UAS, generalised distribution of wheals, concomitant angioedema,increased number of daily attacks and higher pruritus. ASST can be a simple test for detectionof autoreactive CSU in daily practice.

4. Hide $M$, Francis DM, Grattan CE, Hakimi J, Kochan JP, Greaves MW. Autoantibodies against the high-affinity IgE receptor as a cause of histamine release in chronic urticaria. N Engl J Med 1993 Jun 3;328(22):1599-604. https://doi. org/10.1056/NEJM199306033282204

5. Fiebiger $E$, Maurer $D$, Holub $H$, Reininger $B$, Hartmann G, Woisetschlager $M$, et al. Serum IgG autoantibodies directed against the alpha chain of Fc epsilon RI: a selective marker and pathogenetic factor for a distinct subset of chronic urticaria patients? J Clin Invest 1995 Dec;96(6):2606-12. https://doi.org/10.1172/ $\mathrm{JCl} 118325$

6. Konstantinou GN, Asero R, Maurer M, Sabroe RA, Schmid-Grendelmeier P, Grattan CE. EAACI/GA(2)LEN task force consensus report: the autologous serum skin test in urticaria. 
Allergy 2009 Sep;64(9):1256-68. https://doi. org/10.1111/j.1398-9995.2009.02132.x

7. Abd El-Azim M, Abd El-Azim S. Chronic autoimmune urticaria: frequency and association with immunological markers. J Investig Allergol Clin Immunol 2011;21(7):546-50.

8. Alyasin $S$, Hamidi $M$, karimi AA, Amiri A, Ghaffarpasand F, Ehsaei MJ. Correlation between clnical findings and results of autologous serum skin test in patients with chronic idiopathic urticaria. South Med J 2011(Feb);104(2):111-5. https://doi. org/10.1097/SMJ.0b013e31820233fc

9. George $M$, Balachandran C, Prabhu S. Chronic idiopathic urticaria: comparison of clinical features with positive autologous serum skin test. Indian J Dermatol Venereol Leprol 2008;74(2):105-8. https://doi. org/10.4103/0378-6323.39690

10. Vohra S, Sharma NL, Mahajan VK, Shanker V. Clinico-epidemiologic features of chronic urticaria in patients having positive versus negative autologous serum skin test: a study of 100 Indian patients. Indian J Dermatol Venereol Leprol 2011;77(2):156-9. https://doi. org/10.4103/0378-6323.77454

11. Zhong $H$, Song $Z$, Chen W, Li H, He L, Gao T, et al. Chronic urticaria in Chinese population: a hospital-based multicenter epidemiological study. Allergy 2014(Mar);69(3):359-64. https:// doi.org/10.1111/all.12338
12. Asero $R$, Tedeschi A, Lorini $M$, Salimbeni $R$, Zanoletti T, Miadonna A. Chronic urticaria: novel clinical and serological aspects. Clin Exp Allergy 2001 Jul;31(7):1105-10. https://doi. org/10.1046/j.1365-2222.2001.01131.x

13. Godse KV. Autologous serum skin test in chronic idiopathic urticaria. Indian J Dermatol Venereol Leprol 2004;70(5):283-4.

14. Krupashankar DS, Shashikala K, Madala R. Clinical and investigative assessment of patients with positive versus negative autologous serum skin test: a study of 80 South Indian patients. Indian J Dermatol 2012 Nov;57(6):434-8. https://doi. org/10.4103/0019-5154.103061

15. Sabroe RA, Grattan CE, Francis DM, Barr RM, Kobza Black A, Greaves MW. The autologous serum skin test: a screening test for autoantibodies in chronic idiopathic urticaria. $\mathrm{Br}$ J Dermatol 1999 Mar;140(3):446-52. https://doi. org/10.1046/j.1365-2133.1999.02707.x

16. Asero R. Sex differences in the pathogenesis of chronic urticaria. J Allergy Clin Immunol 2003 Feb; 111(2):425-6. https://doi.org/10.1067/ mai. 2003.15

17. Kulthana K, Jiamton S, Gorvanich T, Pinkaew S. Autologous serum skin test in chronic idiopathic urticaria: prevalence, correlation and clinical implications. Asian Pac J Allergy Immunol 2006(Dec);24(4):201-6.

18 Al-Hamamy, Hameed AF, Abdulhadi AS. Autologous Serum skin test in a diagnostic aid in chronic idiopathic urticaria.ISRN Dermatol 2013: 291524. https://doi.org/10.1155/2013/291524 